\title{
Aripiprazole in the treatment of the psychosis
}

\section{prodrome}

\author{
An open-label pilot study
}

SCOTT W. WOODS, ELIZABETH M. TULLY, BARBARA C. WALSH, KEITH A. HAWKINS, JENNIFER L. CALLAHAN, SHUKI J. COHEN, DANIEL H. MATHALON, TANDY J. MILLER and THOMAS H. McGLASHAN

\author{
Background Research studies for the \\ treatment of the putative prodromal \\ phase of psychotic disorders have begun to \\ appear.
}

\begin{abstract}
Aims To obtain preliminary evidence of the short-term efficacy and safety of aripiprazole treatment in people with the psychosis prodrome.
\end{abstract}

Method Fifteen participants meeting prodrome criteria (mean age 17.I years, s.d. $=5.5$ ) enrolled in an open-label, singlesite trial with fixed-flexible dosing of aripiprazole (5-30 mg/day) for 8 weeks.

Results In the mixed-effects repeatedmeasures analysis, improvement from baseline on the Scale of Prodromal Symptoms total score was statistically significant by the first week. No participant converted to psychosis and 13 completed treatment. Neuropsychological measures showed no consistent improvement; mean weight gain was $1.2 \mathrm{~kg}$. Akathisia emerged in 8 participants, but the mean Barnes Akathisia Scale score fell to baseline levels by the final visit. Adverse events were otherwise minimal.

\section{Conclusions Aripiprazole shows a promising efficacy and safety profile for the psychosis prodrome. Placebo- controlled studies are indicated.}

Declaration of interest S.W.W. has received grants from Bristol-Myers Squibb, Janssen, and Eli Lilly.
The prodromal phase of schizophrenic disorders has been recognised since the 19th century (Bleuler, 1911) and the possibility of treatment during the prodromal phase has a history almost as long (Sullivan, 1927). Although some studies have begun to investigate methods to prevent progression from the putatively identified prodromal phase to frank psychosis (Falloon, 1992; McGorry et al, 2002; Morrison et al, 2004; McGlashan et al, 2006), fewer have focused on the acute treatment effects on current symptoms (Woods et al, 2003). Aripiprazole is a relatively new antipsychotic medication with limited liability for weight gain (Marder et al, 2003), whose mechanism of action differs from other antipsychotics in that it is a partial agonist rather than a full antagonist at dopamine $\mathrm{D}_{2}$ receptors (Burris et al, 2002). The overall goal of the present pilot study was to obtain preliminary information about the efficacy and safety of aripiprazole in relieving symptoms that may be prodromal for schizophrenia.

\section{METHOD}

\section{Sample}

Adult participants gave written informed consent and minors gave written informed assent with consent from a parent or guardian. Participants were included if they were treatment-seeking out-patients of 1340 years of age who met diagnostic criteria for a possible prodromal syndrome. People were excluded for any of the following reasons: (a) past or current DSM-IV criteria (American Psychiatric Association, 1994) for any lifetime psychotic disorder; (b) they were judged clinically to have a psychiatric disorder (e.g. mania, depression, attentiondeficit hyperactivity disorder) which could account for the symptoms; (c) they presented with symptoms occurring primarily as sequelae to drug or alcohol use; (d) alcohol or drug misuse or dependence in the past 3 months; (e) use of antipsychotic medication in the previous 3 months; (f) change in dosage of any antidepressant within 6 weeks, stimulant medication within 4 weeks, or mood stabiliser within 4 weeks.

The Criteria of Prodromal Syndromes (COPS; Woods et al, 2001) were used to identify those possibly prodromal. The COPS are based on sub-threshold levels of positive symptoms and operationally define three prodromal syndromes (Yung et al, 1998): attenuated positive symptom syndrome, brief intermittent psychotic syndrome, and genetic risk and recent functional decline syndrome. The COPS and the three syndromes are described in detail elsewhere (Woods et al, 2001; Miller et al, 2003a). Individuals were assessed to determine whether the COPS were met by using the Structured Interview for Prodromal Syndromes (SIPS; Miller et al, 1999). Reliability of the COPS diagnosis of possible prodrome has been excellent when using the SIPS (Miller et al, 2002, 2003a), and patients thus diagnosed are symptomatic (Miller et al, 2003b), functionally impaired (Miller et al, 2003b), cognitively impaired (Hawkins et al, 2004a) and treatment-seeking (Preda et al, 2002).

\section{Study design}

Participants were enrolled between October 2004 and February 2006. The Yale Human Investigation Committee Institutional Review Board approved the protocol. The trial is registered with ClinicalTrials.gov (NCT00237874). This was an open-label study at one site for 8 weeks, followed by an open-label extension phase with monthly follow-up visits to 52 weeks. Findings from the extension phase will be reported subsequently.

\section{Procedure}

During the 1-2 weeks prior to beginning study medication, participants underwent eligibility and neuropsychological examinations. After beginning study medication, participants were scheduled for eight weekly visits.

Dosing followed a fixed-flexible schedule. Initial doses were $5 \mathrm{mg} /$ day aripiprazole; after 1 week, the dose was scheduled for increase to $10 \mathrm{mg} /$ day and after 2 weeks to $15 \mathrm{mg} /$ day, unless adverse effects dictated a slower titration schedule. After the third week, the dose could be increased further to $20 \mathrm{mg} /$ day and if needed to 
$30 \mathrm{mg} /$ day should the person not be responding. Aripiprazole was prescribed as a single daily dose unless there was a reason to divide the dose. The number of milligrams prescribed and the number taken were recorded each day; these data were used to calculate the percentage adherence since the previous visit. Drowsiness was managed initially by switching the timing of the daily dose to bedtime or by dividing the dose. Insomnia was managed initially by switching the timing of the daily dose to early morning. Lorazepam was used to treat insomnia or agitation. Lorazepam or the anticholinergic benztropine was permitted for extrapyramidal symptoms (EPS). Participants continued doses of antidepressant, mood stabiliser, or stimulant medication prescribed before consent but were not permitted to begin or increase dosage of these medications after consent. Individual and family psychosocial interventions with supportive and psychoeducational components were available to each participant.

\section{Assessments}

The primary efficacy measure for the analysis of acute treatment was change over time in the total score of the Scale of Prodromal Symptoms (SOPS; Miller et al, 1999), a 19item scale with items scored 0-6. The interrater reliability has been excellent (Miller $e t$ $a l, 2003 a$ ). Factor analysis supports the validity of the SOPS sub-scales (Hawkins et $a l, 2004 b)$. Treatment response was defined as all five SOPS positive symptom items being rated below the prodromal range (i.e. $\leqslant 2$ ).

Secondary efficacy assessments included the Calgary Depression Scale for Schizophrenia (CDSS; Addington et al, 1990), the Young Mania Rating Scale (YMRS; Young et al, 1978), the Beck Anxiety Inventory (BAI; Beck et al, 1988), the Global Assessment of Functioning Scale (GAF; Hall, 1995), the Heinrichs-Carpenter Quality of Life role functioning sub-scale (HCRF; Heinrichs et al, 1984), and the Social Functioning Scale (SFS; Birchwood et al, 1990).

Neuropsychological assessments included tests of attention and working memory: the Continuous Performance Task (CPT; Cornblatt et al, 1988), identical pairs version, letter number sequencing, N-back, Trails A and B; Stroop Color Word Test for processing speed; Auditory Verbal Learning Task (AVLT; Rey, 1964), using alternate forms for verbal memory; and the Wisconsin Card Sort Test (WCST; Heaton et al, 1993), semantic (category) fluency, and Controlled Oral Word Association (FAS; Spreen \& Benton, 1969) test of phonemic fluency for executive functioning.

Abnormal involuntary movements and EPS were assessed by observation and administration of the Simpson-Angus Scale (SAS; Simpson \& Angus, 1970), the Barnes Akathisia Scale (BAS; Barnes, 1989), and the Abnormal Involuntary Movement Scale (AIMS; Branch, 1975). Safety was also assessed by analysing treatment-emergent adverse events (Systematic Assessment For Treatment-Emergent Events, SAFTEE, specific inquiry method; Levine \& Schooler, 1986), vital signs and weight. Treatmentemergent adverse events were defined as those first occurring or worsening after baseline. All of the above measures were assessed at baseline and weekly thereafter.

\section{Statistical analyses}

For the present report, the time frame was the first 8 weeks after beginning study medication, and the principal outcome measure was the SOPS total score. Analyses were based on the intent-to-treat (ITT) principle. All participants were included in the analysis. The principal outcome measure was addressed using a mixed-effects likelihood-based repeated measures linear model (MMRM, as implemented in SAS PROC MIXED) on post-baseline change scores, using baseline scores as a covariate. For other measures we used $t$-test end-point models at 8 weeks, carrying forward the last observation (LOCF).

\section{RESULTS}

\section{Participants}

A total of 15 participants were enrolled. Demographic and treatment characteristics at baseline are shown in Table 1. All were diagnosed with the common attenuated positive symptom syndrome putative prodrome subtype according to the COPS and none also qualified for either of the other two less common COPS prodromal syndromes. All but two had never received antipsychotic medication prior to participating. One participantt had received antipsychotic for 5 weeks 5 years earlier, and the other for 6 months ending 17 months before baseline, both for indications other than psychosis. Scores for severity of illness at baseline are shown in Table 2. Although there are a dearth of age-specific normative data (our sample with test-retest data has a mean age of 15.5 years, s.d.=1.3), baseline data (Table 3) suggest mild neuropsychological impairment similar to that observed in our previous prodromal sample (Hawkins et al, 2004a). A higher mean AVLT total score was reported for a younger sample by Spreen \& Strauss (1998) and substantially better Trail-Making Part A and Part $B$ performances were reported for a healthy sample aged 15-17 years by Fromm-Auch \& Yeudall (1983).

Thirteen participants completed the 8week study $(87 \%)$. Of the two drop-outs, one completed 48 days on aripiprazole and dropped out because of improvement, feeling medication was no longer needed. The other completed only 8 days on medication and left the study primarily because of sedation after the first $10 \mathrm{mg}$ dose, after having concluded that $5 \mathrm{mg}$ was ineffective after the first week.

\section{Medication}

Prescribed mean (s.d.) aripiprazole doses at weeks $1,2,3,4,5,6,7$ and 8 were $5(0), 9$ (2), 11 (5), 11 (7), 11 (6), 13 (6), 14 (8), and 15 (7) $\mathrm{mg} / \mathrm{day}$, respectively. Final prescribed doses were $5 \mathrm{mg} /$ day ( $n=1$ participant), $10 \mathrm{mg} /$ day $(n=3), \quad 15 \mathrm{mg} /$ day (s.d.=7) $(n=6), \quad 20 \mathrm{mg} /$ day $\quad(n=4) \quad$ and $30 \mathrm{mg} /$ day $(n=1)$. Reported mean (s.d.) percentage adherence with prescribed aripiprazole doses was 94 (13), 92 (22), 98 (4), 96 (8), 97 (7), 95 (10), 98 (5), and 94 (9) at weeks $1,2,3,4,5,6,7$ and 8 respectively. New concomitant medication used after

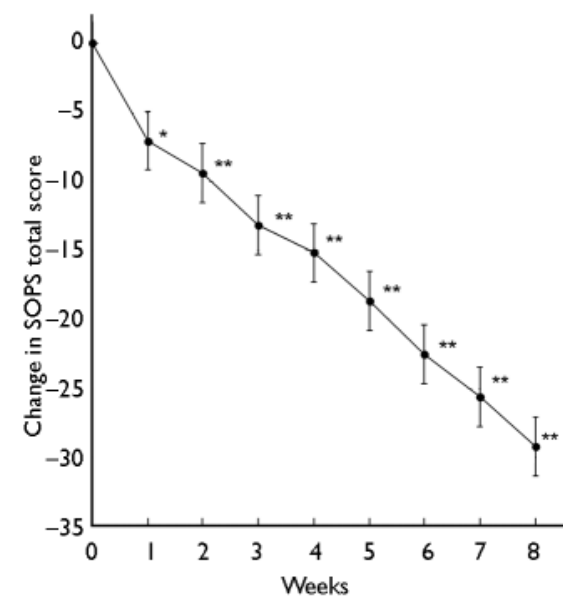

Fig. I Change in Scale of Prodromal Symptoms (SOPS) total score rom baseline with aripiprazole treatment. Mixed-effect repeated-measures modelderived mean and standard error are shown for each time point. $* P<0.05, * * P<0.001$. 
Table I Demographic and treatment data at baseline

\begin{tabular}{lc}
\hline Characteristic & \\
\hline Age, years: mean (s.d.) & $17.1(5.5)$ \\
Male, $n$ (\%) & $8(53)$ \\
White, $n$ (\%) & II (73) \\
Single, $n$ (\%) & $15(100)$ \\
First-degree family history, $n$ (\%) & \\
Psychosis & $3(20)$ \\
Non-psychotic major depression & $6(40)$ \\
Non-psychotic bipolar disorder & I (7) \\
Medication use at baseline', $n$ (\%) & \\
Antidepressants & $7(47)$ \\
Antipsychotics & $0(0)$ \\
Benzodiazepines/hypnotics & $2(13)$ \\
Mood stabilisers & $0(0)$ \\
Stimulants & I (7) \\
Lifetime substance & \\
misuse/dependence, $n$ (\%) & \\
Marijuana & I (7) \\
Other (except nicotine) &
\end{tabular}

I. Patients taking antidepressants, anxiolytics, mood stabilisers, or stimulants were permitted to continue these medications.

2. Includes alcohol, sedatives, opioids, cocaine, hallucinogens and others.

enrollment included benzodiazepine in three participants $(20 \%)$ and anticholinergic medication in 5 participants $(33 \%)$.

\section{Efficacy}

In the mixed-effects model, the effect of time for the SOPS total score change from baseline was statistically significant $(F$ (7) $=9.2, P<0.001$. The reduction in the SOPS total score was statistically significant at each time point (Fig. 1). The LOCF analyses revealed that improvement on the SOPS was statistically significant at endpoint for each of the positive, negative, disorganisation and general symptom subscales (Table 2). The LOCF analyses also revealed significant symptomatic improvement from baseline on the YMRS, CDSS and BAI scales, as well as significant functional improvement on the GAF and HCRF scales (Table 2).

A total of 11 participants met response criteria $(73 \%)$ at week $2(n=1), 3(n=2), 4$ $(n=1), 6(n=4), 7 \quad(n=1)$ and $8 \quad(n=2)$. Response was sustained thereafter until end-point in all but one participant. No participant converted to psychosis. Two non-responders who completed the 8-week course elected not to continue aripiprazole

Table 2 Baseline and change from baseline in severity of illness scores of participants treated with aripiprazole

\begin{tabular}{|c|c|c|c|}
\hline \multirow[b]{2}{*}{ Measure } & \multicolumn{3}{|c|}{ Mean } \\
\hline & Baseline & Change from baseline' & $t(14)^{2}, P$ \\
\hline SOPS Total & $43.3(15.0)$ & $-29.1(12.3)$ & $-9.2<0.001$ \\
\hline Positive & $13.6(3.7)$ & $-10.0(4.1)$ & $-9.4<0.001$ \\
\hline Negative & $12.9(6.9)$ & $-8.2(6.5)$ & $-4.9<0.001$ \\
\hline Disorganisation & $7.1(3.8)$ & $-4.8(3.3)$ & $-5.6<0.001$ \\
\hline General & $9.7(4.2)$ & $-6.1(2.6)$ & $-9.1<0.001$ \\
\hline YMRS total & $4.7(3.7)$ & $-3.9(3.6)$ & $-4.3<0.001$ \\
\hline CDSS total & $4.6(4.5)$ & $-3.8(4.2)$ & $-3.4 \quad 0.005$ \\
\hline BAI total & $14.9(12.3)$ & $-13.1(11.2)$ & $-4.5<0.001$ \\
\hline Current GAF & $4 I .5(6.4)$ & $9.2(5.3)$ & $6.7<0.00$ I \\
\hline HCRF & I4.2(4.7) & $3.2(5.1)$ & $2.3 \quad 0.044$ \\
\hline SFS & $118(30)$ & $-2(2 I)$ & $-0.3 \quad 0.734$ \\
\hline
\end{tabular}

SOPS, Scale of Prodromal Symptoms; YMRS, Young Mania Rating Scale; CDSS, Calgary Depression Scale for Schizophrenia; BAI, Beck Anxiety Inventory; GAF, Global Assessment of Functioning; HCRF, Heinrichs-Carpenter Quality of Life role functioning sub-scale; SFS, Social Functioning Scale.

I. Last-observation-carried-forward values at 8 weeks.

2. $n=14$, d.f. $=13$ for CDSS; $n=13$, d.f. $=12$ for HCRF and SFS.

after 8 weeks; the remaining 11 who completed 8 weeks elected to continue on aripiprazole into the extension phase.

Table 3 shows the results of the neuropsychological testing. Participants improved as a group on two tests of attention and working memory at the significant or trend level (2-digit CPT reaction time, 2-back number correct) but worsened on one other (2-digit CPT performance). They improved as a group on a test of executive functioning (WCST perseverative errors) but worsened on another (semantic fluency). Scores on the remaining 15 tests of attention, working memory, executive functioning, processing speed and verbal memory did not change significantly.

\section{Safety}

One participant discontinued aripiprazole because of adverse events (sedation after 8 days). As determined by the SAFTEE, there were few adverse events of more than mild severity (Table 4). Complaints of adverse events tended to remit over time. Other than the participant who took medication for only 8 days, at the final evaluation an emergent SAFTEE complaint of moderate or greater severity was present in only one participant (nasal congestion).

During treatment, eight participants experienced emergent akathisia demonstrated by increases from baseline on the BAS. Emergent akathisia was managed by slowing dose titration (1), prescribing anticholinergic medication (2), slowing dose titration and prescribing anticholinergic medication (2), slowing dose titration and prescribing benzodiazepine (2) and prescribing anticholinergic medication and then adding benzodiazepine (1). In the context of these management efforts, all participants experiencing emergent akathisia completed treatment, and the emergent akathisia remitted by the final evaluation in six participants. Mean BAS total scores consequently returned to baseline by the end-point evaluation (Table 5). Four participants continued to receive medication for akathisia at the 8-week evaluation.

Little change from baseline to end-point was observed for blood pressure (Table 5). Pulse increased 6 beats per minute on average. There were no significant differences from baseline to end-point on the SAS or AIMS scales (Table 5). Patients gained a mean of $1.2 \mathrm{~kg}$ in weight (Table 5).

\section{DISCUSSION}

The principal finding of the present study was that those meeting criteria for a schizophrenic prodromal syndrome who were treated with aripiprazole improved to a significant degree over an 8-week period on the SOPS and other rating scales. Adverse effects were generally mild and manageable. Important limitations, however, are the small sample size and the use of an uncontrolled, open-label design. 
Table 3 Baseline and change from baseline in neuropsychological functioning measures $(n=10)^{1}$

\begin{tabular}{|c|c|c|c|}
\hline \multirow[b]{2}{*}{ Domain } & Baseline' & Change from baseline ${ }^{1,2}$ & \multirow[b]{2}{*}{$t(9)^{\prime} P$} \\
\hline & & Mean (s.d.) & \\
\hline \multicolumn{4}{|l|}{ Attention and working memory } \\
\hline CPT-IP D-prime (2 digit) & $3.5(0.7)$ & $-0.3(0.4)$ & -2.50 .040 \\
\hline CPT-IP D-prime (3 digit) & $2.3(0.7)$ & $-0.2(0.8)$ & -0.50 .598 \\
\hline CPT-IP D-prime (4 digit) & $1.2(0.5)$ & $-0.2(0.6)$ & -0.80 .452 \\
\hline CPT-IP reaction time (2 digit) $)^{3}$ & $454(39)$ & $31(40)$ & 2.20 .066 \\
\hline CPT-IP reaction time $(3 \text { digit })^{3}$ & 477 (79) & $27(60)$ & 1.30 .249 \\
\hline CPT-IP reaction time (4 digit) $)^{3}$ & $469(48)$ & $-14(64)$ & -0.60 .547 \\
\hline Letter number sequencing & $12.9(4.3)$ & $-0.3(1.7)$ & -0.60 .563 \\
\hline I-back number correct & $47.5(17.9)$ & $-0.8(15.7)$ & -0.10 .896 \\
\hline 2-back number correct & $26.8(16.1)$ & $9.1(10.5)$ & 2.50 .044 \\
\hline Trails $A^{3}$ & $31.9(11.3)$ & $0.3(7.0)$ & 0.10 .890 \\
\hline Trails $\mathrm{B}^{3}$ & $95.9(54.2)$ & $2.3(37.9)$ & 0.20 .858 \\
\hline \multicolumn{4}{|l|}{ Processing speed } \\
\hline Stroop colours & $59.9(15.2)$ & $-0.8(I I .4)$ & -0.20 .829 \\
\hline Stroop words & $82.0(21.0)$ & $-2.1(14.1)$ & -0.50 .648 \\
\hline Stroop colour-word & $35.5(8.5)$ & $0.0(3.5)$ & $0.0 \quad 1.000$ \\
\hline \multicolumn{4}{|l|}{ Verbal memory } \\
\hline AVLT immediate trials sum & 47.7 (II.7) & $0.4(13.5)$ & 0.10 .927 \\
\hline AVLT delay trial & $10.5(2.6)$ & $-0.3(2.4)$ & -0.40 .703 \\
\hline \multicolumn{4}{|l|}{ Executive functioning } \\
\hline WCST perseverative errors ${ }^{3}$ & $10.9(6.4)$ & $3.6(5.2)$ & 2.10 .072 \\
\hline WCST categories completed & $4.0(0.9)$ & $0.0(0.7)$ & $0.0 \quad 1.000$ \\
\hline Semantic fluency & $36.9(10.1)$ & $-4.3(4.0)$ & -3.40 .008 \\
\hline Phonemic fluency & $30.6(8.9)$ & $-1.1(5.1)$ & -0.70 .513 \\
\hline
\end{tabular}

CPT-IP, Continuous Performance Task Identical Pairs version; AVLT, Rey Auditory Verbal Learning Test; WCST, Wisconsin Card Sort Test.

I. $n=\mathbf{9}$ and d.f. $=\mathbf{8}$ for letter number sequencing, Trails $A$ and B, and WCST; $n=8$ and d.f. $=7$ for CPT and N-back.

2. Positive change score and positive $t$ value indicate improvement.

3. Lower scores are better on these measures. The signs for the change scores and $t$ values have therefore been reversed so that positive change scores and positive $t$ values still indicate improvement.

Improvements observed could have been a result of placebo effects or simply the passage of time.

\section{Other studies}

No participants converted to psychosis during the 8-week trial. We would have expected two or three conversions without treatment, based on the placebo group in our previous study (McGlashan et al, 2006). Caution is indicated in comparing our current findings with our historical placebo group, however, because it is possible that people volunteering for an open-label trial could differ from those volunteering for a placebo-controlled study (Woods et al, 2005).

This is the second report to our knowledge to focus on an acute pharmacological treatment of symptoms that can be
Table 4 Treatment-emergent adverse events in participants with prodromal symptoms treated with aripiprazole

\begin{tabular}{ll}
\hline SAFTEE items' & $n(\%)$ \\
\hline Irritability & $5(33)$ \\
Increased appetite & $4(27)$ \\
Sedation & $3(20)$ \\
Insomnia & $2(13)$ \\
Nervousness & $2(13)$ \\
Memory impaired & $2(13)$ \\
Sensory perception impaired & $2(13)$ \\
Saliva increased & $2(13)$ \\
Libido decreased & $2(13)$ \\
Excessive sweating & $2(13)$ \\
\hline
\end{tabular}

SAFTEE, Systematic Assessment for Treatment-

Emergent Events.

I. Proportion of participants endorsing adverse events as determined by the SAFTEE at the moderate level or higher at any time point and representing an increase over baseline. Adverse events endorsed by only one participant are not shown.

sample demography were similar. Improvement on GAF, as well as on mania and depression measures, was also more robust in the open-label study of aripiprazole than we had previously observed in a marked study of olanzapine.

Weight gain with aripiprazole also compared favourably with our previous experience with olanzapine. Participants treated with olanzapine gained a mean of $4.5 \mathrm{~kg}$ over 8 weeks in LOCF analyses, whereas the aripiprazole mean weight gain in this study was $1.2 \mathrm{~kg}$ (Table 5) despite being exposed to drug for a higher proportion of the 8 weeks. This degree of weight gain was comparable to that observed in previous short-term studies of aripiprazole (mean $0.71 \mathrm{~kg} v$. no change for placebo; Marder et al, 2003).

Akathisia, on the other hand, was more problematic with aripiprazole than it had been with olanzapine, leading to higher rates of benztropine prescription. However, benztropine, or in some cases slowing of dose titration or benzodiazepine prescription, was effective in managing this adverse effect, so that by the final evaluation the net effect of treatment on akathisia ratings was similar to what we had observed with olanzapine.

Adherence with prescribed doses was relatively high in this short-term analysis as measured by participant report at each visit. Adherence was similar to or higher than with olanzapine in our masked study, although the latter used a somewhat more 
Table 5 Baseline and change from baseline in vital signs, weight, extrapyramidal symptoms and abnormal involuntary movements

\begin{tabular}{|c|c|c|c|}
\hline \multirow[b]{2}{*}{ Measure } & Baseline & Change from baseline' & \multirow[b]{2}{*}{$t(14)^{2} P$} \\
\hline & \multicolumn{2}{|c|}{ Mean (s.d.) } & \\
\hline \multicolumn{4}{|c|}{ Blood pressure, $\mathrm{mmHg}$} \\
\hline Systolic & $120(12)$ & $3(12)$ & 1.00 .338 \\
\hline Diastolic BP & $65(10)$ & I (9) & 0.50 .635 \\
\hline Pulse, beats/min & 69 (II) & $6(14)$ & 1.40 .181 \\
\hline Weight, kg & $67.4(18.1)$ & $\mathrm{I} .2(2.0)$ & 2.20 .049 \\
\hline SAS total score & $0.6(0.9)$ & $-0.3(0.8)$ & -1.50 .136 \\
\hline BAS total score & I.2(I.3) & $-0.7(1.6)$ & -1.60 .136 \\
\hline AIMS total score & $0.1(0.5)$ & $-0.1(0.5)$ & -1.00 .335 \\
\hline
\end{tabular}

SAS, Simpson-Angus Scale; BAS, Barnes Akathisia Scale; AIMS, Abnormal Involuntary Movement Scale.

I. Last-observation-carried-forward values at 8 weeks.

2. $n=13($ d.f. $=12)$ for weight and $n=14($ d.f. $=13)$ for blood pressure and pulse.

rigorous pill-count method of monitoring adherence (Woods et al, 2003). As for most studies, surreptitious non-adherence cannot be excluded. Future studies should continue to assess adherence in this population.

Our neuropsychological findings show little consistent effect of aripiprazole over 8 weeks. Among the 20 results reported, a few tests did show improvement, but others showed a decline, with most suggesting little change. Considering tests that did change, within domain of function there appeared to be little consistency in direction. Certainly the limited sample size would have predisposed our study to low power to detect possible real effects; on the other hand, some gains would be expected on the basis of prior test exposure. In the only previous study, to our knowledge, on neuropsychological effects of aripiprazole (Kern et al, 2006), 169 participants with chronic schizophrenic psychosis underwent neuropsychological examinations before and 8 weeks after random assignment to aripiprazole or olanzapine. Aripiprazole-treated participants improved significantly over 8 weeks on a general cognitive factor on which loaded our letter number sequencing, verbal fluency and trail-making tasks. Our failure to find consistent improvement on these measures could relate to our small sample or to our participants being less impaired and having less room to improve. Aripiprazole-treated participants improved significantly in the previous study (Kern et al, 2006) on a verbal learning factor on which loaded a test similar to our AVLT but without use of alternative forms. Our failure to find consistent improvement on verbal memory could relate to the small sample, studying prodromal $v$. chronic illness, or, our use of a counterbalanced alternate form for the repeat verbal learning list, which may have prevented the confounding of measurement of new verbal learning by familiarity with the word list carried over from the baseline testing (practice effect; Hawkins \& Wexler, 1999; Hawkins et al, 2004c). In the previous study (Kern et al, 2006) aripiprazole-treated participants improved but not significantly on an executive functioning factor on which loaded our WCST tasks. These findings were similar to ours with WCST perseverative errors.

\section{Dosing}

We paid close attention to the dosing of aripiprazole in the current study. Although some investigators have emphasised that patients meeting prodrome criteria can be managed with antipsychotic doses which are lower than those used in chronic schizophrenia (Falloon, 1992; McGorry et al, 2002), there had been some evidence in our previous study that olanzapine had been used at too low a dose, especially in the first month (McGlashan et al, 2006). In the present study we employed a recommended fixed dose titration by the end of the second week to $15 \mathrm{mg} /$ day, the minimal consistently effective aripiprazole dose for patients with chronic schizophrenia (Woods, 2003), unless the prescriber had a specific reason to deviate from this schedule. The $15 \mathrm{mg} /$ day dose was also that most commonly employed in a recent pilot study of aripiprazole in first-episode schizophrenia (Brown et al, 2003). Initial doses were $5 \mathrm{mg} /$ day, in keeping with guidelines for initiation of aripiprazole in adolescents/children who weigh $50-70 \mathrm{~kg}$ (Findling et al, 2004).

\section{Implications}

Our current findings are relevant to discussions of the ethics of intervention research in people who appear prodromal. Prodrome research studies thus far have primarily focused on preventing the development of psychosis. Although this is certainly an important goal, ethical issues are raised because some participants will be false-positives who have no personal opportunity to benefit if benefit is defined solely as prevention. The current data suggest that people carrying a risk of progression to psychosis can receive not only the possibility of a preventive benefit but also a treatment benefit 'on average' from intervention. The prospect of treatment benefit on average is generally considered sufficient to justify exposure to some treatment risk in other illnesses. Thus the current data strengthen the argument that intervention studies can be ethical with people who appear prodromal.

Although our findings suggest that people who meet prodrome criteria benefit when prescribed aripiprazole, the present results contribute to what is only the beginning of the process of establishing a standard of care for such people. The sample size in our study was small and we had no control group, placebo or otherwise. Future placebo-controlled studies with more participants are needed before recommendations can responsibly be made regarding routine treatment. The present findings suggest that aripiprazole is a promising candidate for such studies.

\section{ACKNOWLEDGEMENTS}

This study was supported by an investigator-initiated grant (S.W.W.) from Bristol-Myers Squibb.

\section{REFERENCES}

Addington, D., Addington, J. \& Schissel, B. (1990) A depression rating scale for schizophrenics. Schizophrenia Research, 3, 247-25I.

American Psychiatric Association (1994) Diagnostic and Statistical Manual of Mental Disorderes (4th edn) (DSM-IV). APA.

Barnes, T. R. (1989) A rating scale for drug-induced akathisia. British Journal of Psychiatry, 154, 672-676.

Beck, A. T., Epstein, N. Brown, G., et al (1988) An inventory for measuring clinical anxiety: psychometric 
properties. Journal of Consulting \& Clinical Psychology, 56, 893-897.

Birchwood, M., Smith, J., Cochrane, R., et al (1990) The Social Functioning Scale. The development and validation of a new scale of social adjustment for use in family intervention programmes with schizophrenic patients. British journal of Psychiatry, 157, 853-859.

Bleuler, E. (19II) Dementia Praecox or the Group of the Schizophrenias. International Universities Press.

Branch, P. R. (1975) Abnormal involuntary movement scale (AIMS). Early Clinical Drug Evaluation Unit Intercom, 4, 3-6.

Brown, D., McEvoy, J., Saha, A. R., et al (2003) Aripiprazole in patients with first episode schizophrenia. Schizophrenia Research, 60, 274.

Burris, K. D., Molski, T. F., Xu, C., et al (2002) Aripiprazole, a novel antipsychotic, is a high-affinity partial agonist at human dopamine D2 receptors. Journal of Pharmacology and Experimental Therapeutics, 302. 38I-389.

Cornblatt, B. A., Risch, N. J., Faris, G., et al (1988) The Continuous Performance Test Identical Pairs Version (CPT-IP). I. New findings about sustained attention in normal families. Psychiatry Research, 26 223-238.

Falloon, I. R. H. (1992) Early intervention for first episodes of schizophrenia: a preliminary exploration. Psychiatry, 55, 4-15.

Findling, R., Blumer, J., Kauffman, R., et al (2004) Pharmacokinetic effects of aripiprazole in children and adolescents with conduct disorder. International Journal of Neuropsychopharmacology, 7, S440.

Fromm-Auch, D. \& Yeudall, L.T. (1983) Normative data for the Halstead-Reitan Neuropsychological Tests. Journal of Cliical Neuropsychology, 5, 221-238.

Hall, R. C. (1995) Global assessment of functioning. A modified scale. Psychosomatics, 36, 267-275.

Hawkins, K. A. \& Wexler, B. E. (1999) California Verbal Learning Test: practice effects in a schizophrenia sample. Schizophrenia Research, 39, 73-78.

Hawkins, K. A., Addington, J., Keefe, R. S. E., et al (2004a) Neuropsychological status of subjects at high risk for a first episode of psychosis. Schizophrenia Research, 67, II5-122.

Hawkins, K. A., Quinlan, D., Miller, T. J., et al (2004b) Factorial structure of the scale of prodromal symptoms. Schizophrenia Research, 68, 339-347.

Hawkins, K. A., Dean, D. \& Pearlson, G. D. (2004c) Alternative forms of the Rey Auditory Verbal Learning Test: a review. Behavioural Neurology, 15, 99-107.

Heaton, R. K., Chelune, G. J., Talley, J. L., et al (1993) Wisconsin Card Sorting Test, Manual. Psychological Assessment Resources.

Heinrichs, D. W., Hanlon, T. E. \& Carpenter, W. T., Jr (1984) The Quality of Life Scale: an instrument for

SCOTT W. WOODS, MD, Yale University School of Medicine, New Haven, Connecticut, USA; ELIZABETH M. TULLY, MD, University of New Mexico School of Medicine, Albuquerque, New Mexico, USA; BARBARA C. WALSH, PhD, KEITH A. HAWKINS, PsyD, JENNIFER L. CALLAHAN, PhD, SHUKI J. COHEN, PhD, DANIEL H. MATHALON, PhD, MD, TANDY J. MILLER, PhD (deceased), THOMAS H. McGLASHAN, MD, Yale University School of Medicine, New Haven, Connecticut, USA

Correspondence: Dr Scott Woods, 34 Park Street, New Haven, CT 06519, USA. Email scott.woods@yale.edu

rating the schizophrenic deficity syndrome. Schizophrenia Bulletin, 10, 388-398.

Kern, R. S., Green, M. F., Cornblatt, B. A., et al (2006) The neurocognitive effects of aripiprazole: an open-label comparison with olanzapine.

Psychopharmacology, 187, 312-320.

Levine, J. \& Schooler, N. R. (1986) Strategies for analyzing side-effect data from SAFTEE - a workshop held Fall 1985 in Rockville, Maryland. Psychopharmacology Bulletin, 22, 343-381.

Marder, S. R., McQuade, R. D., Stock, E., et al (2003) Aripiprazole in the treatment of schizophrenia: safety and tolerability in short-term, placebo-controlled trials. Schizophrenia Research, 61, 123-136.

McGlashan, T. H., Zipursky, R. B., Perkins, D., et al (2006) Randomized double-blind clinical trial of olanzapine versus placebo in patients prodromally symptomatic for psychosis. American Journal of Psychiatry, 163, 790-799.

McGorry, P. D., Yung, A. F., Phillips, L. J., et al (2002) Randomized controlled trial of interventions designed to reduce the risk of progression to first-episode psychosis in a clinical sample with subthreshold symptoms. Archives of General Psychiatry, 59, 921-928.

Miller, T. J., McGlashan, T. H., Woods, S. W., et al (1999) Symptom assessment in schizophrenic prodromal states. Psychiatric Quarterly, 70, 273-287.

Miller, T. J., McGlashan, T. H., Rosen, J. L., et a (2002) Prospective diagnosis of the prodrome for schizophrenia: preliminary evidence of interrater reliability and predictive validity using operational criteria and a structured interview. American journal of Psychiatry, 159, 863-865

Miller, T. J., McGlashan, T. H., Rosen, J. L., et a (2003a) Prodromal assessment with the Structured Interview for Prodromal Syndromes and the Scale of Prodromal Symptoms: predictive validity, inter-rater reliability, and training to reliability. Schizophrenia Bulletin, 29, 703-715

Miller, T. J., Zipursky, R. B., Perkins, D. O., et al (2003b) A randomized double blind clinical trial of olanzapine vs placebo in patients at risk for being prodromally symptomatic for psychosis: II. Baseline characteristics of the prodromal sample. Schizophrenia Research, 6I, 19-30.
Morrison, A. P., French, P., Walford, L., et al (2004) Cognitive therapy for the prevention of psychosis in people at ultra-high risk. Randomized controlled trial. British Journal of Psychiatry, 185, 291-297.

Preda, A., Miller, T. J., Rosen, J. L., et al (2002) Treatment histories of patients with a syndrome putatively prodromal for schizophrenia. Psychiatric Services, 53, 342-344.

Rey, R. A. (1964) L'examen clinique en psychologique. Presses Universitaires de France.

Simpson, G. M. \& Angus, J.W. (1970) A rating scale for extrapyramidal side effects. Acta Psychiatrica Scandinavica, Supplementum, 212, 11-19.

Spreen, O. \& Benton, A. L. (1969) Neurosensory Center Comprehensive Examination for Aphasia (NCCEA). University of Victoria Neuropsychology Laboratory.

Spreen, O. \& Strauss, E. (1998) A Compendium of Neuropsychological Tests: Administration, norms, and commentary (2nd edn). Oxford University Press.

Sullivan, H. S. (1927) The onset of schizophrenia American Journal of Psychiatry, 7, 105-134.

Woods, S. W. (2003) Chlorpromazine equivalent dose for the newer atypical antipsychotics. Journal of Clinical Psychiatry, 64, 663-667.

Woods, S. W., Miller, T. J. \& McGlashan, T. H. (200I) The prodromal patient: both symptomatic and at risk. CNS Spectrums, 6, 223-232.

Woods, S. W., Breier, A., Zipursky, R. B., et al (2003) Randomized trial of olanzapine vs placebo in the symptomatic acute treatment of patients meeting criteria for the schizophrenic prodrome. Biological Psychiatry, 54, 453-464.

Woods, S. W., Gueorguieva, R.V., Baker, C. B., et al (2005) Control group bias in randomized atypical antipsychotic medication trials for schizophrenia. Archives of General Psychiatry, 62, 96I-970.

Young, R. C., Biggs, J.T., Ziegler, V. E., et al (1978) A rating scale for mania: reliability, validity and sensitivity. British Journal of Psychiatry, I33, 429-435.

Yung, A. R., Phillips, L. J., McGorry, P. D., et al (1998) Prediction of psychosis. A step towards indicated prevention of schizophrenia. British Journal of Psychiatry 172 (suppl. 33), 14-20. 